\title{
Effects of the FOXO3a rs 4946936 Gene Polymorphism on the FOXO3a Transcription Factor in Chronic Granulocytic Leukemia Patients
}

\author{
Shinta Oktya Wardhani ${ }^{1 *}$ (D) Hani Susianti ${ }^{2}$ (D) Pudji Rahayu ${ }^{3}$, Yuyun Prabowowati Yueniwati $^{4}$ (D) \\ ${ }^{1}$ Department of Internal Medicine, Division of Medical Hematology-Oncology, Faculty of Medical, Brawijaya University, \\ Dr. Saiful Anwar General Hospital Malang, Malang, Indonesia; ${ }^{2}$ Department of Clinical Pathology, Faculty of Medical, Brawijaya \\ University, Dr. Saiful Anwar General Hospital Malang, Malang, Indonesia; ${ }^{3}$ Department of Ear Nose Throat, Faculty of Medical, \\ Brawijaya University, Dr. Saiful Anwar General Hospital Malang, Malang, Indonesia; ${ }^{4}$ Department of Radiology, Faculty of \\ Medical, Brawijaya University, Dr. Saiful Anwar General Hospital Malang, Malang, Indonesia
}

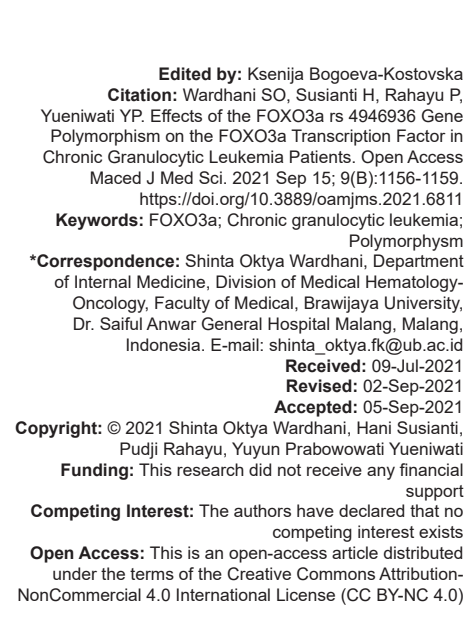

\section{Introduction}

FOXO3a plays an important role in the maintenance of leukemic stem cells. In chronic myelocytic leukemia, CD34+ cells BCR-ABL expression leads to an increase in FOXO3a in the cytoplasm (transcriptionally inactive), whereas in normal CD34+ cells, FOXO3a is detected predominantly in the nucleus. Inhibition of BCR-ABL by tyrosine kinase inhibitor (TKI) reduces the phosphorylation of the transcription factor Foxo3a, promotes localization of Foxo $3 a$ in the nucleus and restores transcriptional activity [1], [2]. A study by Pellicano et al. in 2014 showed that the administration of TKI dasatinib for $24 \mathrm{~h}$ could increase in the relocation of Foxo1 and Foxo3a from the cytoplasm to the nucleus. This study also proved that after the exposure to TKI dasatinib, in addition to an increase in the relocation of Foxo3a to the intranuclear, there was also an increase in the expression of genes that down target Foxo3a intra nucleus ATM, CDKN1c/p57 and Bcl6 which plays a role in increasing the apoptosis of these cells [2].

In the last decade, many studies have shown that genetic factors influence the response to malignancy therapy. In that sense, current therapies are mostly directed at personalized therapy (personalized medicine). FOXO3a single nucleotide polymorphism has a lot of influence on the level of the Foxo3a transcription factor and is associated with several diseases such as vitiligo, chronic obstructive pulmonary disease, bronchial asthma, severe malaria, thyroid cancer, and acute lymphoblastic leukemia, but there has been no study that linked single nucleotide polymorphisms with response therapy [3], [4], [5], [6], [7]. 
Several genes that regulate important proteins in cellular processes such as fertilization, growth, cell cycle, response to stress and oncogenesis are regulated in the translational stage and these settings are mostly found in the untranslated region (UTR), so that an abnormality in this region are usually linked with various disease, such as FOXO3a rs4946936 gene polymorphism found in 3'UTR. Pprevious studies have shown that $\mathrm{FOXO} 3 \mathrm{a}$ gene polymorphisms were associated with several malignancies, such as acute lymphoblastic leukemia, thyroid cancer, and hepatocellular carcinoma (Sionov, Vlahopoulos and Granot, 2015). 3'UTR plays a role in the process of translation, localization and stability of mRNA.

This study was aimed to investigate the effect of the FOXO3a rs4946936 gene polymorphism on the Foxo3a transcription factor in chronic granulocytic leukemia (CGL) patients receiving Imatinib mesylate therapy.

\section{Method}

This is cross-sectional study in CGL patients with positive Bcr-ABL. The aim of this study was to prove the effect of the $\mathrm{FOXO} 3 a$ gene polymorphism rs4946936 on the Foxo3a transcription factor. The inclusion criteria used in this study was positive Bcr-Abl patients aged more than 18 years old who had received imatinib mesylate therapy for at least 6 months. While the exclusion criteria were patients with leukopenia (leukocytes <4000/uL), obstructive pulmonary disease and rheumatoid arthritis, patients with acute lymphoblastic leukemia and patients with vitiligo. This study was approved by the ethics department of Dr. Saiful Anwar Hospital with ethical number: 400/146/K.3/302/2019. The data were analyzed using SPSS 24 software. In the descriptive sample, different tests were used to find conclusions. Meanwhile, to find the effect of the FOXO3a rs 4946936 gene on the therapeutic response, a regression test was used. Results with $p$ value $<0.05$ were considered significant.

\section{examination \\ FOXO3a rs4946936. single polymorphism}

The FOXO3a rs4946936 gene polymorphism was carried out by DNA quantification using a NanoDrop ${ }^{\text {TM }}$ One spectrophotometer (Thermo Fisher Scientific Inc., Wilmington, USA), Measurement of DNA concentration and purity by spectrophotometry. PCR (polymerase chain reaction) examination using CFX 96 TouchTM Real-Time PCR (Bio-Rad, USA). Primer MyTaq HS Mix, 2X, (Bioline, Meridian BioscienceTM, USA) FOXO3a. Restriction fragment length polymorphism was examined with the CFX96 TouchTM
Real-Time PCR Detection System (Bio-Rad, USA) using a restriction enzyme: Sfcl (New England Biolabs, USA) Foxo3a F: 5'-GGGTCCTGAGAACTTCTGAGT-3' Foxo3a R:5 '-GACATTCTGTAAGACATTCTGCCT-3'. The TT genotype was characterized by 224bp sized bands, the TC genotype was characterized by 224 , 152 , and 72 bp sized bands while the CC genotype was characterized by 152 and 72 bp size bands (Figure 1).

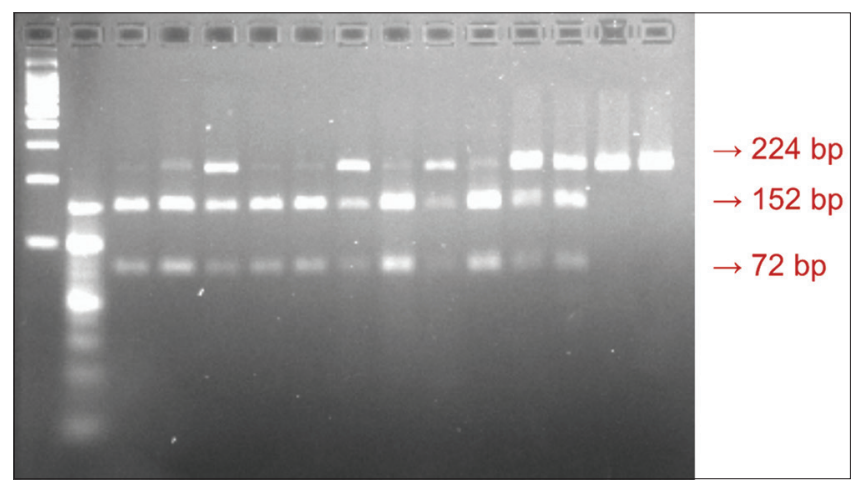

Figure 1: A visualization sample of digestion result using restriction enzyme

Examination of serum Foxo3a levels using the ELISA method was taken by using the Cusabio kit (pg/ $\mathrm{mL}$ ) with the procedure according to the instructions from the company (manufacture). Foxo3a levels were calculated from the standard curve and expressed in units of $\mathrm{pg} / \mathrm{mL}$.

\section{Data analysis}

The research data were analyzed using the SPSS version 24 software program calculations for Windows. Baseline data characteristics of the data were analyzed using the comparative method, where in this study, the Kruskall-Walls test was used. While the parametric test uses the Pearson correlation method and linear regression. Correlation test was used to find the relationship between $\mathrm{FOXO} 3 a$ gene polymorphisms and the transcription factor $\mathrm{FOXO} 3 \mathrm{a}$. While the regression test was used to find the effect of the polymorphism with the transcription factor FOX3a.

\section{Results}

Based on the Table 1, it can be seen that from 60 samples, the average leukocyte of the sample as a whole is 52389.68. In data analysis using the different test, the $p=0.013$ was obtained, so it can be concluded that there is a significant difference between the results of the leukocyte laboratory and the polymorphism. While the average blast sample as a whole is 8.40 . In the data analysis using the different test, the $p=0.000$ was obtained, so it can be concluded that there is a 
Table 1: Baseline characteristics samples

\begin{tabular}{|c|c|c|c|c|c|c|c|c|c|}
\hline \multirow[t]{3}{*}{ Characteristics } & \multicolumn{8}{|c|}{ Gene FOXO3a Polymorphism } & \multirow[t]{3}{*}{$p$-value } \\
\hline & \multicolumn{2}{|l|}{$\mathrm{CC}$} & \multicolumn{2}{|l|}{ TC } & \multicolumn{2}{|l|}{ TT } & \multicolumn{2}{|l|}{ Total } & \\
\hline & $\mathrm{F}$ (mean) & $\%(\mathrm{Sd})$ & $\mathrm{F}$ (mean) & $\%(\mathrm{Sd})$ & $\mathrm{F}$ (mean) & $\%(\mathrm{Sd})$ & $\mathrm{F}$ (mean) & $\%(\mathrm{Sd})$ & \\
\hline \multicolumn{10}{|l|}{ Sex } \\
\hline Male & 11 & $18.30 \%$ & 15 & $25.00 \%$ & 5 & $8.30 \%$ & 31 & $51.70 \%$ & \multirow[t]{2}{*}{0.992} \\
\hline Female & 10 & $16.70 \%$ & 14 & $23.30 \%$ & 5 & $8.30 \%$ & 29 & $48.30 \%$ & \\
\hline Age & (43.62) & (11.75) & $(41.9)$ & (12.53) & $(43.4)$ & (14.18) & $(10.12)$ & $(4.04)$ & 0.877 \\
\hline \multicolumn{10}{|l|}{ Spleen } \\
\hline S3 & 4 & $6.70 \%$ & 4 & $6.70 \%$ & 0 & $0.00 \%$ & 8 & $13.30 \%$ & \multirow[t]{4}{*}{0.455} \\
\hline S4 & 6 & $10.00 \%$ & 7 & $11.70 \%$ & 4 & $6.70 \%$ & 17 & $28.30 \%$ & \\
\hline S5 & 5 & $8.30 \%$ & 13 & $21.70 \%$ & 5 & $8.30 \%$ & 23 & $38.30 \%$ & \\
\hline s6 & 6 & $10.00 \%$ & 5 & $8.30 \%$ & 1 & $1.70 \%$ & 12 & $20.00 \%$ & \\
\hline Hemoglobin & (11.59) & $(4.18)$ & $(10.9)$ & (3) & (10.12) & $(4.04)$ & (11.01) & (3.6) & 0.559 \\
\hline Leukocyte & (25698.1) & $(56533.51)$ & $(42165.55)$ & (94168.6) & (138092) & (162617.05) & (52389.68) & (104285.9) & $0.013^{*}$ \\
\hline Thrombocyte & (218047.62) & (102640.38) & (423241.38) & (963616.32) & (306900) & (183752.52) & (332033.33) & (676914.46) & 0.575 \\
\hline Blast & (6.14) & (5.51) & (5.83) & (7.94) & (20.6) & (17.04) & (8.4) & (10.71) & $0.000^{*}$ \\
\hline
\end{tabular}

significant difference between the results of the blast laboratory and the polymorphism.

There were 3 polymorphisms of the FOXO3a gene, namely CC polymorphism, TC polymorphism, and TT polymorphism with a $p=0.026$ and an $r$ of 0.287 , so it can be concluded that there is a significant relationship between the FOXO3a gene polymorphism and the Foxo3an transcription factor with a sufficient correlation value (Table 2). In the regression test between the FOXO3a gene polymorphisms and the transcription factor FOXO3a, the $p$ value was 0.029 and the $B$ value was -0.294 (Table 3 ). This means that it has a negative and significant effect on the Foxo3a transcription factor variable. The negative regression coefficient explains that the TT polymorphism in the FOXO3a Gene Polymorphism will be able to significantly reduce the Foxo3a transcription factor.

Table 2: The correlation between FOXO3a rs4946936 polymorphism gene and FOX3a transcription factor

\begin{tabular}{lllllll}
\hline FOXO3a polymorphysm gene & \multicolumn{3}{c}{ FOXO3a } & p-value (r) \\
\cline { 2 - 3 } & & F & & Mean & Std & \\
\hline CC & 21 & 35 & 77.34 & 47.64 & $0.026(0.287)$ \\
TC & 29 & 48.3 & 55.71 & 50.86 & \\
TT & 10 & 16.7 & 36.46 & 46.64 & \\
\hline
\end{tabular}

\section{Discussion}

The data on the characteristics of our research participants showed statistically significant differences in the mean number of leukocytes between the CC, TC and TT genotypes of the FOXO3a rs 4946936 gene. The CC genotype (referent genotype) had the lowest number of leukocytes compared to the TC and TT genotypes. The results of the study by Hamed et al. in 2019 showed different results from ours, namely there was an insignificant difference in the mean number of leukocytes in the TET2 gene polymorphism rs34402524 in the LGK population $(p=0.07)$. Different results were also reported by Muddathir et al. in 2019 which showed that there was no difference in the mean number of leukocytes in the GSTT1 and GSTM1 polymorphisms $(p=0.254$ and $p=0.797)[8]$.

The data on the characteristics of our study subjects showed statistically significant differences in the mean number of Blast cells between the $\mathrm{CC}, \mathrm{TC}$ and TT genotypes of the FOXO3a rs 4946936 gene. The CC genotype had the lowest number of blast cells compared to the TC and TT genotypes. Different results were shown in the study of Hamed et al. in 2019. In Hamed's study, there was no significant difference in the number of blast cells in the TET2 gene polymorphism [9].

Table 3: The effect of the FOXO3a rs4946936 polymorphism gene on FOXO3a transcription

\begin{tabular}{lllll}
\hline Variable & B & Count & p-value & Note \\
\hline FOXO3a gene polymorphism & -0.294 & -2.241 & 0.029 & Significant \\
\hline
\end{tabular}

In the last two decades, advances in gene sequencing have been able to document gene sequence variations between individuals, implying that phenotypic diversity is formed by $0.5 \%$ of epigenetic modifications. These sequence differences arise due to insertions, deletions and single nucleotide polymorphisms. When present in genes, SNPs can have an impact on mRNA splicing, nucleo-cytoplasmic export, protein stability and translation, modification of protein activity and protein content. Studies by Ozel Turkcu et al. in 2014 and Kalemci et al. in 2016 showed that FOXO3a rs 4946936 gene polymorphism had an effect on the levels of the Foxo3a transcription factor. In our study, CGL patients with the CC genotype FOXO3a rs4946936 had the highest serum Foxo3a levels compared to the TC and TT genotypes. This result was consistent with a study by Kalemci et al. in 2016 which found that the CC genotype COPD patient population had the highest plasma Foxo3a level compared to the TC and TT genotypes [3], [4].

The difference of Foxo3a levels in different genotypes could be influenced by several factors that affect translation due to changes in mRNA translation at the ' ' and 3' UTR locations which could affect protein levels. The factors of these changes include the presence of a cap or cover at the 5' end, the formation of a secondary structure, the presence of several open reading frames, the presence of multiple 4 AUGs, Internal Ribosome Entry Sites or ribosome entry points, sequence position AUG and polyadenylation motifs and signals due to all of the above factors regulate protein synthesis by causing changes in mRNA stability, ribosomal axes, circulation and translation [10]. 
The difference in Foxo3a levels in the CC genotype in our study is in accordance with our in silico study, in which we found a difference in the free energy level of the CC genotype FOXO3a rs4946936 with the TT genotype $(-320.80 \mathrm{Kcal} / \mathrm{mol}$ vs. $-301.10 \mathrm{Kcal} / \mathrm{mol})$. The existence of this free energy difference is likely to give a difference in the stability of the mRNA. This is in accordance with research from Uddin which showed that low free energy levels were associated with the mRNA structure of various genes that would increase protein synthesis [11]. The research of Salari et al. strengthens the evidence from Uddin's study that various diseases were associated with single nucleotide polymorphisms. It is showed that most of these diseases are associated with the presence of polymorphisms in UTR so that they did not affect the formation of certain amino acids or proteins, but this polymorphism could alter the function and structure of mRNA which would eventually affect the expression, stability, translation regulation, and changes in mRNA structure and protein synthesis [12]. From the in silico study, it was found that the CC genotype had more negative free energy than TT, so that the Foxo protein produced is higher, in accordance with the results of the higher Foxo3a protein content in the CC genotype.

\section{Conclusion}

There was a significant correlation between the FOXO3a rs4946936 gene polymorphism and the FOX3a transcription factor. The FOXO3a gene polymorphism of the TT genotype has a negative effect on the FOXO3a transcription factor. In addition, the TT genotype of the FOXO3a gene polymorphism rs4946936 was the most effective in reducing the transcription factor $\mathrm{FOXO} 3 \mathrm{a}$.

\section{References}

1. Pellicano F, Holyoake T. Assembling defenses against therapyresistant leukemic stem cells: Bcl6 joins the ranks. J Exp Med. 2011;208(11):2155-8. https://doi.org/10.1084/jem.20112087 PMid:22025499

2. Pellicano F, Sinclair A, Holyoake T. In search of CML stem cells deadly weakness. Curr Hematol Malig Rep. 2011;6(2):82-7. https://doi.org/10.1007/s11899-011-0085-y

PMid:21373837

3. Turkcu UO, Tekin NS, Edgunlu TG, Celik SK, Oner S. The association of $\mathrm{FOXO} 3 \mathrm{~A}$ gene polymorphisms with serum FOXO3A levels and oxidative stress markers in vitiligo patients. Gene. 2014;536(1):129-34. https://doi.org/10.1016/ s0378-1119(13)00039-5

PMid:24333267

4. Kalemci S, Edgunlu TB, Turkcu UO, Sakalh E, Cetin ES, Zeybek $\mathrm{A}$, et al. $\mathrm{FOXO}$ a gene polymorphism and serum FOXO3a levels in patients with chronic obstructive pulmonary disease and healthy controls: Effects of genetic polymorphism in chronic obstructive pulmonary disease. Smyrna Tıp Derg. 2013;1:1-5. https://doi.org/10.5114/kitp.2014.45682

5. El Rifai NM, Al-Wakeel H, Osman HM, El Taweel RH. FOXO3a gene polymorphism and bronchial asthma in Egyptian children. Egypt J Pediatr Allergy Immunol. 2019;17(1):31-6. https://doi. org/10.21608/ejpa.2019.41535

6. Nguetse CN, Kremsner PG, Velavan TP. FOXO3A regulatory polymorphism and susceptibility to severe malaria in Gabonese children. Immunogenetic. 2014;67(2):67-71. https://doi. org/10.1007/s00251-014-0816-z

PMid:25421486

7. Rohlen N, Doring C, Hansmann ML, Grunwald F, Vorlander C, Bechstein WO, et al. FOXO3a polymorphism rs4946936 and its interaction with Vitamin $D$ in differentiated thyroid cancer. Exp Clin Endocrinol Diabetes. 2014;122(3):84. https://doi. org/10.1055/s-0034-1372101

8. Muddathir A, Abdallah E, Khabour O, Abdelgader R, Elgari M Age- and gender-independent association of glutathione S-transferase null polymorphisms with chronic myeloid leukemia. Bosn J Basic Med Sci. 2019;19(4):350-4. https://doi. org/10.17305/bjbms.2019.4176

PMid:30995900

9. Obied M, Al-Tu'ma F, Al-Jameel H. Role of polymorphism (rs1024611) in monocyte chemoattractant protein-1 gene in diabetic foot ulcer of Iraqi patients. Gene Rep. 2019;17:100502. https://doi.org/10.1016/j.genrep.2019.100502

10. Sionov RV, Vlahopoulos SA, Granot Z. Regulation of BIM in health and disease. Oncotarget. 2015;6(27):23058-134. https:// doi.org/10.18632/oncotarget.5492

PMid:26405162

11. Uddin SN, Majunder A, Islam KK, Hossain SA, Sarker PK Minimum free energy based evaluation of mRNAs secondary structures constructed by 18 clinically significant exonic single nucleotide polymorphisms (SNPs) and haplotypes of 5 missense SNPs of RB1 gene. Am J Biochem Biotechnol. 2015;11(4):191-9. https://doi.org/10.3844/ajbbsp.2015.191.199

12. Salari R, Kimchi-Sarfaty C, Gottesman MM, Przytycka TM Sensitive measurement of single-nucleotide polymorphisminduced changes of RNA conformation: Application to disease studies. Nucleic Acids Res. 2012;41(1):44-53. https://doi. org/10.1093/nar/gks1009

PMid:23125360 